\title{
NEW DIAGNOSTICS OF LASER GENERATED SHOCKS
}

\author{
C. Stehlé ${ }^{1}$, N. Champion ${ }^{1}$, P.A. Delattre ${ }^{1}$, U. Chaulagain ${ }^{1}$, M. Kozlová ${ }^{2}$, \\ J. $\operatorname{Nejdl}^{2}$, J. Dostál ${ }^{2}$, M. Krus ${ }^{2}$, J. Larour ${ }^{3}$, F. Suzuki-Vidal ${ }^{4}$, O. Acef ${ }^{5}$, \\ P. Barroso ${ }^{6}$ and J.P. Chièze ${ }^{7}$
}

\begin{abstract}
Strongly radiative shocks are characterized by an ionization front induced by the shock wave. The role played together by opacity and geometry is critical for the physics of these shock waves. Moreover, radiation is an obvious way of probing these shock waves, either by self-emission or by probe absorption. These aspects will be illustrated by recent experimental results obtained at the iodine PALS (Prague Asterix Laser System) facility.
\end{abstract}

\section{Introduction}

Our motivation is to understand the physics of shock waves occurring during the early stages of stellar evolution, when young stars accrete matter from their surrounding circumstellar environment. The observed excess luminosity and X-ray spectral signatures, resulting from these accretion shocks, are used to estimate, for instance, the accretion rate. These flows have complex structures, and drastic modelling approximations have been used so far, oversimplifying their geometry or neglecting the coupling between radiation and hydrodynamics. Thus, accretion rates are uncertain by up to a factor of 10 and the picture of the basic proces in star formation remains rather sketchy. The accretion flows on young stars are believed to follow cylindrical funnels connecting the stellar photosphere to the disk along the lines of the magnetic field (Bouvier et al. 2006). However, there is not a clear

${ }^{1}$ LERMA, Observatoire de Paris, UPMC, CNRS, 5 place J. Janssen, 92195 Meudon, France

2 Institute of Physics of ASCR, Na Slovance 2, 18221 Prague 8, Czech Republic

3 LPP, École Polytechnique, UPMC, CNRS, 91128 Palaiseau, France

4 The Blackett Laboratory, Imperial College, Prince Consort Road, London SW7 2AZ, UK

5 SYRTE, Obs. Paris, UMPC, CNRS, 61 avenue de l'Observatoire, 75014 Paris, France

${ }^{6}$ GEPI, Obs. Paris, Paris Diderot, CNRS, 61 avenue de l'Observatoire, 75014 Paris, France

7 IRFU/Service d'Astrophysique, CEA-Saclay, 91191 Gif-sur-Yvette, France 
consensus on the location of the shock wave, which could be either in the chromosphere or in the photosphere, and this could have an impact on the coupling between radiation and the flow, leading to the shock structure. The modelling requires state of the art multidimensional hydrodynamical and radiative transfer codes. In this context, experiments allow studying these hypersonic flows in regimes similar to the astrophysical cases, with the help of numerical simulations, which, in return, are used for the modelling of astrophysical shocks.

Strong radiative shocks can be generated in gases using high energy lasers. Depending on the opacity, the photons emitted from the shock front might be reabsorbed by the cold gas in which the shock propagates, generating an ionisation wave, also called a radiative precursor (Fleury et al. 2002; Bouquet et al. 2004; Reighart et al. 2006; Doss et al. 2009). The complexity of the physics implied, which results from the radiation effects, is enhanced by the non-ideal effects in the gas (e.g. equation of state) and by the geometrical effects (Michaut et al. 2004; Leygnac et al. 2006; Gonzalez et al. 2006).

\section{Experiment}

Despite the large number of experiments in the last decade, probing the whole shock structure, i.e. radiative precursor, shock front and post-shock, remains challenging. Moreover, the study of the radiative signatures of the shocks is still at infancy (Stehlé et al. 2010). Thus, the experiments presented here aim at testing the feasibility of two new diagnostics (Stehlé et al. 2012): instantaneous imaging of the shock structure with an auxiliary XUV laser at $21.2 \mathrm{~nm}$, and a photometric study of space and time resolved plasma self-emission which will also be useful to follow the shock chronometry.

The experimental setup (Fig. 1) consists of two beams from the PALS iodine laser (hereafter AUX and MAIN), with a pulse duration of 0.3 ns. The first beam (AUX, $60 \mathrm{~J}, 10^{14} \mathrm{~W} / \mathrm{cm}^{2}$ ) drives the shock wave at $\sim 60 \mathrm{~km} / \mathrm{s}$ inside a target filled with Xenon gas (with a pressure of 0.3 bar). The targets used for the shock generation consist on a shock tube of section $0.4 \times 0.4 \mathrm{~mm}^{2}$, adapted to the AUX beam section on the target. The length of the tube is $6 \mathrm{~mm}$. The tube is closed by a foil (piston), on which the laser beam is focused, which consists of a film of polystyren ( $\mathrm{CH}, 10 \mu \mathrm{m}$ thick) followed by a thin $(0.5 \mu \mathrm{m}$ thick $)$ layer of gold. The laser ablation of the polystyren generates the shock wave, which propagates through the gold layer and the Xenon gas. The gold layer acts as a radiation shield, preventing the X-rays induced by the ablation to preheat the Xenon inside the cell. For the XUV backlighting, as for plasma self-emission diagnostics, the shock tube has lateral $150 \mathrm{~nm}$ thick $\mathrm{Si}_{3} \mathrm{~N}_{4}$ windows.

The second laser beam (MAIN, $500 \mathrm{~J}$ ) is focused on a Zn slab-target to generate a XUV laser beam at $21.2 \mathrm{~nm}$ (Rus et al. 2002). This XUV beam (hereafter called XRL beam) is used as a backlighter of the shock wave, with a delay of 20 ns after the AUX beam reaches the piston, allowing an instantaneous imaging of the shock. The XRL beam propagates through the shock-target, is reflected by a spherical $\mathrm{Mo} / \mathrm{Si}$ multilayer mirror, passes through a pinhole located in the focal plane of 


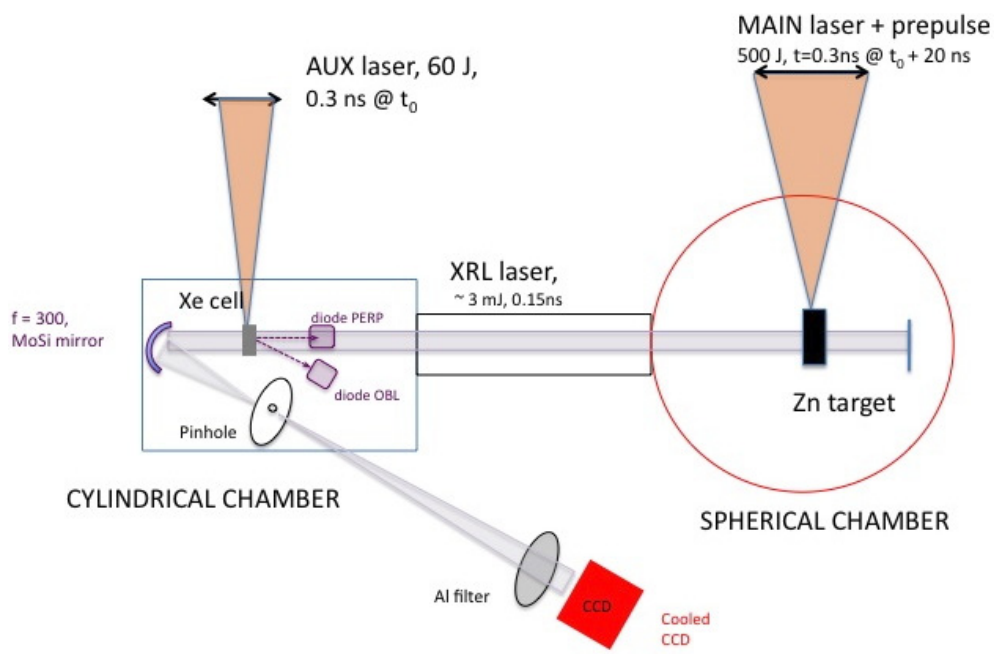

Fig. 1. Experimental setup showing the AUX and delayed MAIN infrared laser beams, which drive respectively the shock wave in cylindrical chamber and the XRL laser in the spherical chamber. The XUV imaging and diode diagnostics are also shown.

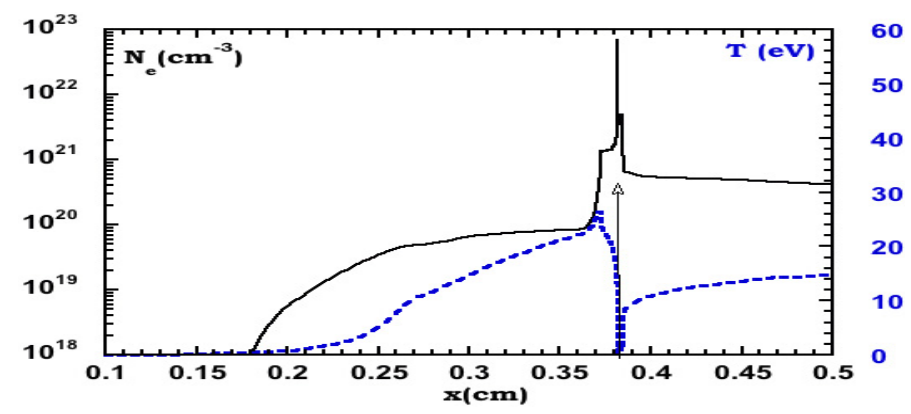

Fig. 2. Variations of $N_{e}$ (left scale, full line) and $T$ (right scale, dashed line) versus distance at $20 \mathrm{~ns}$. The position of the piston is initially at $\mathrm{z}=0$. The position of the $\mathrm{Au}-\mathrm{Xe}$ interface is indicated by a vertical arrow.

this mirror and reaches a cooled CCD camera located at the position of the image of the shock tube through this mirror (magnification of 8.4) (Stehlé et al. 2012). The monochromatic radiography with the XRL offers several advantages: the large number of photons, a collimated beam, and the possibility to probe dense matter up to a fraction of the corresponding electron critical density (i.e. $2.5 \times 10^{24} \mathrm{~cm}^{-3}$ at $21 \mathrm{~nm}$ ), thus the dense part of the shock. The structure of the plasma (Xe, Au, $\mathrm{CH}$ ), expected from 1D simulations (Ramis et al. 1998) is reported in Figure 2. The Figure 3 shows the ratio of two recorded images of the tube illuminated by the XRL laser, with and without shock wave. The expected spatial resolution is 


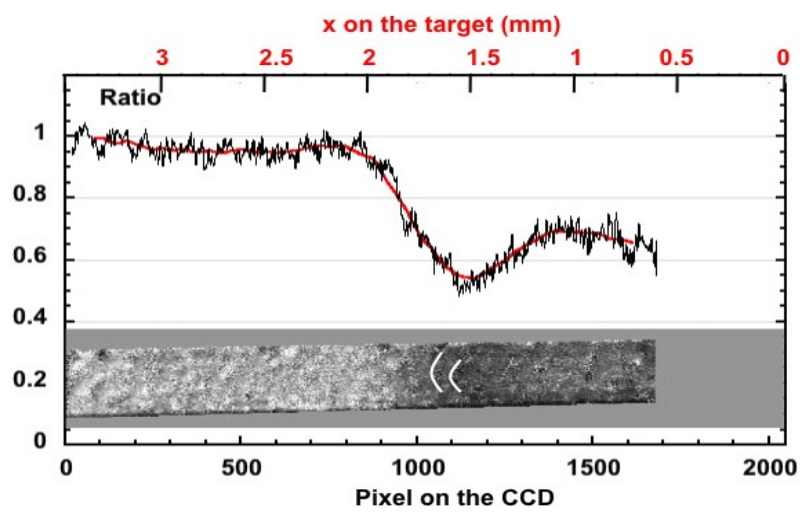

Fig. 3. Image of the shock wave under backlighting with the XRL laser. The shock is moving from right to left. The position $\mathrm{x}=0 \mathrm{~mm}$ corresponds to the initial location of the piston. Values of the transmission, normalized to 1 on the left, are also given.

of about $50 \mu \mathrm{m}$ and the edges of the $\mathrm{Si}_{3} \mathrm{~N}_{4}$ windows are indeed very sharp on these records (see Stehlé et al. 2012). However, the poor quality of XUV imaging mirrors induces parasitic static dark structures in the images covering zones of about $70 \mu \mathrm{m}$ on the target and which remain in the computed absorption figure. The absorption is maximum for pixel 1100 (i.e. $1.5 \mathrm{~mm}$ from the initial position of the piston). The image remains dark for positions larger than 1100 pixels. Taking into account the delay of 20 ns between XRL and AUX lasers, the record is compatible with a shock velocity of about $70 \mathrm{~km} / \mathrm{s}$. We observe a significant absorption between pixels 800 and 1100 which we expect to come from the heated Xenon in the precursor. However, due to the insufficient quality of the XUV image, the structure remains difficult to interpret. Two high-speed $1-\mathrm{mm}^{2}$ Silicon bare diodes were located perpendicularly to the longitudinal axis of the shock tube to record the time-dependent self-emission of the plasma at $2.2 \mathrm{~mm}$ from the initial position of the piston. The spatial resolution is ensured by adequate Tungsten slits which, for a shock velocity of $60 \mathrm{~km} / \mathrm{s}$, result in a time window of about $5-7 \mathrm{~ns}$. We observe (Fig. 4) a strong peak at time $\mathrm{t}=0$, which is attributed to stray light during the intense interaction between AUX and the $\mathrm{CH}-\mathrm{Au}$ piston foil. The signals from the diodes increases after $20 \mathrm{~ns}$, reaching a maximum at $\sim 40 \mathrm{~ns}$, which is consistent with a shock velocity of $\sim 55 \mathrm{~km} / \mathrm{s}$.

\section{Conclusions}

We present the first experimental study of radiative shock waves in gases by simultaneous XRL imaging and spatially-resolved plasma self-emission. The XRL backlighting allows probing not only the shock front but also part of the radiative precursor. Concerning the diode diagnostics, the present results open a door to more detailed studies of the plasma self-emission. Foreseen studies will improve 


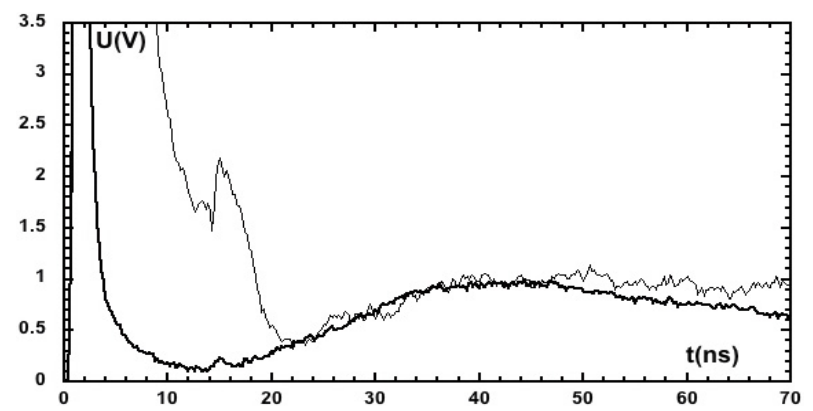

Fig. 4. Signal recorded by the two high speed Si diodes.

these results by increasing the quality of the multi-layer mirror used for XRL imaging to analyze more precisely the structure and the expected multidimensional geometry of these shocks.

The work was supported by LASERLAB access program, French ANR (grant 08-BLAN-026307), PICS4343 of CNRS, University Pierre et Marie Curie, Observatoire de Paris, the Academy of Sciences of the Czech Republic (Project M100100911), the Czech Ministry of Education, Youth and Sports (Projects 7E08099 and 7E09092), and the Czech Science Foundation (Grant 202/08/1734).

\section{References}

Stehlé, C., Kozlova, M., Larour, J., et al., 2012, Optics Comm., 285, 64

Stehlé, C., Gonzalez, M., Kozlova, M., et al., 2010, LPB, 28, 253

González, M., Audit, E., \& Stehlé, C., 2009, A\&A, 497, 27

Doss, F.W., Robey, H.F., Drake, R.P., \& Kuranz, C.C., 2009, Phys. Plasmas, 16, 112705

Reighard, A.B., Drake, R.P., Dannenberg, K.K., et al., 2006, Phys. Plasmas, 13, 082901

Leygnac, S., Boireau, L., Michaut, C., et al., 2006, Phys. Plasmas, 13, 113301

González, M., Stehlé, C., Audit, E., et al., 2006, Laser Part. Beams, 24, 535

Bouvier, J., Alencar, S.H.P., Harries, T.J., et al., 2006, in Protostars and Planets V, ed.

B. Reipurth, D. Jewitt \& K. Keil [arXiv:astro-ph/0603498]

Michaut, C., Stehlé, C., Leygnac, S., et al., 2004, Eur. Phys. J. D, 28, 381

Bouquet, S., Stehlé, C., Koenig, M., et al., 2004, PRL, 92, 225001

Rus, B., Mocek, T., Präg, A.R., et al., 2002, PRA, 66, 063806

Fleury, X., Bouquet, S., Stehlé, C., et al., 2002, Laser Part. Beams, 20, 63

Ramis, R., Schmalz, R., \& Meyer-Ter-Vehn, J., 1998, CPC, 49, 475 